\title{
Genetic variation within and relationships among populations of Asian goats (Capra hircus)
}

\begin{abstract}
Genetic variation at 59 protein coding loci (16 polymorphic) and 25 microsatellite loci was analysed for 11 indigenous south-east Asian goat populations, and the Australian feral population, to determine the magnitude of genetic differentiation and the genetic relationships among the populations. Significant deviations from Hardyï Weinberg equilibrium were detected in one or more populations for eight of the nine protein loci with codominant alleles, and for microsatellites for all except the two Sri Lankan populations and for all but four loci. For both marker types, average inbreeding coefficients (FIS) were exceptionally high. Heterogeneity of deviations from Hardyï Weinberg equilibrium for the microsatellites showed no differences for among loci within populations as compared with among populations within loci. For protein loci, however, the former was higher, indicating selection affecting allele frequencies at some loci. The variance among protein loci was significantly higher than among microsatellite loci, further indicating selection at some protein loci. There was significant differentiation among populations for both protein and microsatellite loci, most likely reflecting the geography of south-east Asia, and the presumed spread of goats throughout the region. Phylogenies derived from pair-wise genetic distance estimates show some similar clustering for the microsatellite and protein based trees, but bootstrap support was generally low for both. A phylogeny based on the combined set of 38 protein and microsatellite loci showed better consistency with geography and higher bootstrap values. The genetic distance phylogeny and the Weitzman diversity tree derived from microsatellite data showed some identical clusters, and both identified the Ujung Pandang and Australia populations as contributing most to overall genetic diversity.
\end{abstract}

Keyword: Biochemical polymorphism; Microsatellites; Genetic distance; Phylogeny; Inbreeding 